\title{
Interventions to Improve Antibiotic Prescribing in Upper Middle Income Countries: A Systematic Review of the Literature 1990 - 2009
}

\author{
Verica Ivanovska ${ }^{1,2}$, Kathleen Anne Holloway ${ }^{3}$ \\ ${ }^{1}$ Utrecht University - Utrecht Institute for Pharmaceutical Sciences, Division of Pharmacoepidemiology and Clinical Pharmacology, Utrecht,

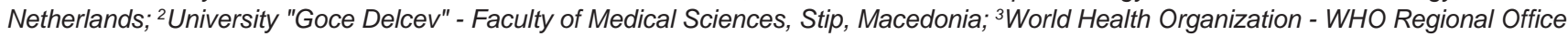 \\ South East Asia, Delhi, India
}

\begin{abstract}
Citation: Ivanovska V, Holloway KA. Interventions to Improve Antibiotic Prescribing in Upper Middle Income Countries: A Systematic Review of the Literature 1990 - 2009. Maced J Med Sci. 2013 Mar 15; 6(1):84-91. http:/dx doi.org/10.3889/ MJMS.1857-5773.2012.0268.

Key words: antibiotics; appropriate use prescribing practices; indicators; interventions; primary health care; medical dociors; system review; upper middle-income countries.

Correspondence: Mrs. Verica Ivanovska. Radika 5-2-3, Skopje 1000, Republic of Macedonia. EMail: vericaivanovska@hotmail.com

Received: 17-Jan-2012; Revised: 02-Nov-2012; Accepted: 03-Nov-2012; Online first: 27-Nov-2012

Copyright: @ 2012 Ivanovska V. This is an openaccess article distributed under the terms of the Creative Commons Attribution License, which permits unrestricted use, distribution, and reproduction in any medium, provided the orig author and source are credited.

Competing Interests: The author have declared that no competing interests exist.
\end{abstract}

\begin{abstract}
Background: Inappropriate antibiotic use is a global public health problem with serious consequences, including antimicrobial resistance. In response, countries have to take comprehensive action with interventions that improve antibiotic use at various levels. Several reviews have evaluated interventions on antibiotic prescribing practices in different healthcare settings.
\end{abstract}

Objective: To identify interventions targeting antibiotic prescribing by medical doctors in primary health care in upper middle-income countries and to assess intervention effectiveness.

Methods: We undertook systematic literature review of studies for the period 1990-2009. Studies had to report quantitative data on antibiotic prescribing by primary care doctors using medicines use indicators. Intervention effects were based on data from intervention studies with valid study designs.

Results: Eight studies describing ten interventions met all the inclusion criteria. We found that single educational interventions targeting all diseases had low or no impact on percentages of patients prescribed antibiotics. Greater impact on antibiotic prescribing was achieved by multifaceted interventions focusing on specific diseases.

Conclusion: The limited evidence on interventions from upper middle-income countries has produced results similar to other reviews. More concerted commitment is needed to monitor antibiotic prescribing regularly and to conduct well designed evaluations of interventions.

\section{Introduction}

Since the introduction of penicillin in the 1940s, antibiotics have been essential for the treatment of many bacterial infections. Meanwhile, antibiotics have become subject to many overuse and misuse prescribing practices. They have been unnecessarily prescribed for viral infections, against which they have no effect $[1,2]$. Similarly, when diagnoses had not been accurately made, broad-spectrum antibiotics have been prescribed because the causative micro-organism has been unknown [1]. Widespread irrational patterns include antibiotic use in acute diarrhoea or in upper respiratory tract infections (URTI) with predominantly viral aetiology [3-12].

A World Health Organization (WHO) review has shown increase in antibiotic use over the last 20 years in developing and transitional countries, reaching $50 \%$ of prescriptions in primary health care. Half of antibiotics were prescribed inappropriately or in underdosage. Over two-thirds of all URTI cases received antibiotics 
unnecessarily, while less than $80 \%$ of pneumonia cases were treated with appropriate antibiotics [3]. Data from the European Surveillance of Antibiotic Consumption demonstrated large variations in outpatient antibiotic consumption across 25 European countries [11]. Antibiotic overprescribing has been demonstrated even in European countries with lower rates of antibiotic use, such as the Netherlands [12].

Inappropriate antibiotic use entails an increased risk of side effects, high costs, and accelerated emergence, selection and spread of resistant bacteria [11,13-15]. Microorganisms become resistant to affordable and effective first-line antibiotics, which leads to inadequate treatment of the infection $[1,2,16]$. Unfortunately, while resistance to older antibiotics is increasing, development of new generations of antibiotics is not promising [17].

Therefore, growing antimicrobial resistance associated with inappropriate antibiotic use is of great public health concern globally $[3,12,18,19]$. In response, numerous policies and guidelines promoting appropriate use of available antibiotics have been introduced internationally [20-27]. However, combating inappropriate use of medicines requires comprehensive action with interventions that improve medicines use at different levels [28-31].

Several systematic reviews have summarized effects of different types of interventions targeting health providers' prescribing practices. Published studies have focused on different aspects on medicines use, various diseases and health care settings in both developed and developing countries [3, 29, 32-35]. Despite diversity, their results showed similar findings. Most interventions have been educational in nature, but multi-faceted interventions, involving educational and managerial components, have been more effective than those employing only one strategy [3, 29, 32-35].

Our research focused on antibiotic prescribing interventions in upper middle-income countries (UMICS). This particular income group is selected to represent settings comparable to the Republic of Macedonia, classified as UMIC by the World Bank [36]. Our review is expected to inform future national strategies on appropriate antibiotic use in the Republic of Macedonia.

This research aims to: (1) undertake literature review to identify interventions targeting antibiotic prescribing by medical doctors in primary health care in UMICs, (2) describe intervention studies with valid study designs, and (3) assess intervention effectiveness.

\section{Methodology}

\section{Search strategy and criteria}

The systematic search was conducted using published articles and unpublished reports for the period 1990 - 2009. The sources included: the International Network for the Rational Use of Drugs (INRUD) Bibliography on medicines use [37], Embase, PubMed, World Health Organization (WHO) and international agencies' archives on medicines programmes, primary care and child health. Study inclusion criteria were: an 'intervention' aiming to 'improve antibiotic prescribing practices' by 'medical doctors' conducted in a 'primary care' setting in an 'upper middle-income country'. Studies had to report quantitative data on antibiotic prescribing by primary care doctors using medicines use indicators. According to the World Bank (WB) 2010 classification, UMICs had Gross National Income (GNI) per capita in the range $\$ 3,976$ - $\$ 12,275$, and included 54 countries from 6 regions in 2010 [36].

\section{Criteria for methodological adequacy}

Only intervention studies using randomized control trials (RCT), pre-post measurements with a control group (PP\&C), and interrupted time series with or without a comparison group (TS\&C, TS-C) were considered to have valid study designs.

Other key aspects of methodological quality taken into consideration were the overall patients sample size and the number of healthcare facilities included in the intervention surveys. Studies involving less than 100 cases or 5 health facilities were excluded from the review.

\section{Classification of interventions}

The commonly used interventions were broken down into the following categories and subcategories, described below: [3, 28-31]

1. Provider education (Formal education, Seminars and trainings, Academic detailing);

2. Printed materials (Formulary lists, Treatment guidelines, Newspapers and bulletins);

3. Consumer education (Media - TV and radio, Printed material, Home visits);

4. Managerial strategies (Clinical supervision systems, Audit and/or feedback, Drug utilization review, Drug Therapeutic Committees); 
5. Community Case Management (Training/ supervision of community members to provide treatment for certain diseases, Medicines supply to trained layperson);

6. Group process strategies (Review, Drug Therapeutic Committees, Management, training and planning activities);

7. Regulatory interventions (Professional licensing, Registration, Practice laws);

8. Economic strategies (Prescription fee and consultation fee, fee per drug item, Revolving drug fund, Health insurance);

9. Essential Medicines Programme (Essential drugs list, Structured prescribing forms, Generic substitution, Prior authorization, Medicines supply).

\section{Measuring study effects and effect size}

Main outcome measures used to assess changes in antibiotic use were changes in medical doctors' antibiotic prescribing. The antibiotic outcomes included certain WHO/INRUD indicators [38] and WHO $\mathrm{IMCl}$ indicators [39], but excluded DDD measures and patients' adherence indicators. The antibiotic prescribing outcomes (prescribing indicators) were as follows: (1) Percentage of patients prescribed antibiotics, (2) Percentage of viral upper respiratory tract infections (URTI) treated with antibiotics, (3) Percentage of pneumonia cases treated with appropriate antibiotics.

The intervention effects were based on data from intervention studies with valid study designs. The method that summarised interventions' effects compared improvement in each antibiotic use outcome as reported by individual authors. In all cases other than for pneumonia, the desired improvement was a reduction in use. However, for pneumonia, the goal was to increase the use of appropriate antibiotics in patients suspected of having the disease. The effect sizes for all outcome measures were calculated as follows:

\section{Effect Size $=(\%$ Post-\% Pre) Intervention (\%Post-\% Pre) Control}

When intervention studies reported multiple postintervention assessments, the last post-intervention data point was used to calculate study effects. For time series with no control, the effect size was the net difference between the last post intervention value and the pre intervention value.
Large impact was defined as more than $30 \%$ improvement, moderate impact as $10-30 \%$ of improvement, and low or no impact as less than $10 \%$ of improvement in a targeted outcome relative to control.

Where available, we accompanied our calculated effect sizes by information on results' variability and statistical significance, as stated in the original papers. Original details on confidence intervals or $p$ values were intended to show results strength and precision, and demonstrate the quality of corresponding studies.

\section{Data extraction}

Data were extracted using designed data extraction sheet with following information: (1) Literature source, (2) Demographic details (country, years of measurement, health care setting and ownership, patient age, target disease), (3) Methodological details (study design, number of facilities and cases), (4) Intervention components and types, and (5) Outcome antibiotic measures (indicator types) and intervention effect sizes.

\section{Results}

\section{Literature review outcome}

We initially screened 60 potential studies from 22 UMICs that included interventions on antibiotic prescribing in primary health care. However, only 12 studies had valid study designs according to our methodological criteria. Additionally, four studies were excluded because they reported antibiotic prescribing by health workers other than medical doctors, such as paramedical workers, nurses, pharmacists and trained lay persons. Finally, eight studies (describing ten interventions) met all the inclusion criteria and were included in the review [40-47].

\section{Description of intervention studies}

Seven studies were retrieved from published articles, while only one was an unpublished report from WHO archives. Studies were distributed over three World Bank geographical regions: East Asia and Pacific (China and Malaysia), Middle East and North Africa (Iran) and Latin America (Cuba, Mexico and Ecuador). No studies with valid design were identified from Europe/Central Asia and Sub-Saharan Africa.

Six studies were carried out after year 2000 , and two in the early 1990s. Six surveys were conducted in 
public primary health care centres (PHC), and only two in outpatient departments within public hospitals. No study with valid study design has been conducted in the private sector. Half of the interventions targeted one specific health condition: acute respiratory illness (ARI), while the rest included all illnesses from primary healthcare settings. Six studies analysed patients of all ages, and two included only children under 5 years old.

In terms of methodology employed, one study was a randomized controlled trial, and seven studies were pre-post-measurements with a comparison group. Table 1 gives a comprehensive summary of all reviewed studies and their references, including demographic and methodological study characteristics and intervention types.

\section{Intervention types}

In total, ten interventions of six types were employed to optimise antibiotic prescriptions by medical doctors. Five of them were single-component, and five

Table 1: Description of included interventions.

\begin{tabular}{|c|c|c|c|}
\hline Ref & $\begin{array}{l}\text { Demography I } \\
\text { Targets }\end{array}$ & Methodology & Intervention type(s) \\
\hline$\# 40$ & $\begin{array}{l}\text { China } 2000 \\
\text { Public hospital } \\
\text { All ages } \\
\text { All diseases }\end{array}$ & $\begin{array}{l}\text { PP\&C } \\
7 \text { facilities } \\
1500 \text { cases }\end{array}$ & $\begin{array}{l}\text { (1) Provider and consumer } \\
\text { education } \\
\text { (2) Economic strategies, } \\
\text { (3) EML }\end{array}$ \\
\hline $\begin{array}{l}\# 41 \\
\text { (a) }\end{array}$ & $\begin{array}{l}\text { Cuba } 1991 \\
\text { Public PHC } \\
\text { Child<5yrs } \\
\text { ARI }\end{array}$ & $\begin{array}{l}\text { PP\&C } \\
40 \text { households } \\
1600 \text { cases }\end{array}$ & (1) Consumer education \\
\hline $\begin{array}{l}\# 41 \\
\text { (b) }\end{array}$ & $\begin{array}{l}\text { Cuba } 1991 \\
\text { Public PHC } \\
\text { Child }<5 y \text { rs } \\
\text { ARI } \\
\end{array}$ & $\begin{array}{l}\text { PP\&C } \\
40 \text { households } \\
1600 \text { cases }\end{array}$ & $\begin{array}{l}\text { (1) Provider education } \\
\text { (2) Managerial strategy }\end{array}$ \\
\hline $\begin{array}{l}\# 41 \\
\text { (c) }\end{array}$ & $\begin{array}{l}\text { Cuba } 1991 \\
\text { Public PHC } \\
\text { Child<5yrs } \\
\text { ARI } \\
\end{array}$ & $\begin{array}{l}\text { PP\&C } \\
40 \text { households } \\
1600 \text { cases }\end{array}$ & $\begin{array}{l}\text { (1) Provider and consumer } \\
\text { education } \\
\text { (2) Managerial strategy }\end{array}$ \\
\hline$\# 42$ & $\begin{array}{l}\text { Ecuador } 2001 \\
\text { Public hospitals } \\
\text { Child <5yrs } \\
\text { ARI } \\
\end{array}$ & $\begin{array}{l}\text { PP\&C } \\
8 \text { facilities }\end{array}$ & $\begin{array}{l}\text { (1) Provider education } \\
\text { (2) Managerial strategy }\end{array}$ \\
\hline$\# 43$ & $\begin{array}{l}\text { Iran } 2006 \\
\text { Public PHC } \\
\text { All ages } \\
\text { All diseases } \\
\end{array}$ & \begin{tabular}{|l|} 
RCT \\
112 facilities \\
13480 cases
\end{tabular} & (1) Provider education \\
\hline$\# 44$ & $\begin{array}{l}\text { Iran year N/A } \\
\text { Public PHC } \\
\text { All ages } \\
\text { All diseases } \\
\end{array}$ & \begin{tabular}{|l|} 
PP\&C \\
80 MDs \\
1096861 cases
\end{tabular} & (1) Provider education \\
\hline$\# 45$ & $\begin{array}{l}\text { Iran } 2002 \\
\text { Public PHC } \\
\text { All ages } \\
\text { All diseases }\end{array}$ & \begin{tabular}{|l} 
PP\&C \\
51 MDs \\
4516 cases
\end{tabular} & (1) Provider education \\
\hline$\# 46$ & $\begin{array}{l}\text { Malaysia } 2004 \\
\text { Public PHC } \\
\text { All ages } \\
\text { All diseases + ARI }\end{array}$ & \begin{tabular}{|l} 
TS-C- \\
9facilities \\
23180 cases
\end{tabular} & (1) Provider education \\
\hline$\# 47$ & $\begin{array}{l}\text { Mexico1990 } \\
\text { Public PHC } \\
\text { All ages } \\
\text { ARI } \\
\end{array}$ & $\begin{array}{l}\text { PP\&C } \\
18 \text { facilities } \\
504 \text { cases }\end{array}$ & $\begin{array}{l}\text { (1) Provider education } \\
\text { (2) Managerial strategy }\end{array}$ \\
\hline
\end{tabular}

PHC - Primary Healthcare Centre, ARI - Acute Respiratory Infection, MD - Medical Doctors, PP\&C - Pre-post with control group, RCT - Randomized control trial, TS-C - Time series without control group, EML - Essential Medicines List. were multi-components. The interventions were as follows:

1. Provider education (4 studies);

2. Consumer education (1 study);

3. Provider education and supervision (1 study);

4. Provider education with group process strategies - peer review committee (1 study) and pharmacy committee (1 study);

5. Provider and consumer education with supervision (1 study); and

6. Multifaceted intervention consisting of educational, economic, and essential drug list strategies (1 study).

All of the interventions involved educational components and half of them consisted of only one educational component (provider or consumer education). The other half involved educational interventions combined with managerial ones.

\section{Intervention effectiveness}

Table 2 gives details of the intervention components, study outcomes, statistical significance of the results in the original studies, and calculated effect sizes.

Table 3 summarizes the intervention impact on antibiotic indicator values in descending order by the degree of effect.

The positive sign (+) illustrates positive intervention impacts, such as reduced antibiotic use in all patients, reduced antibiotic use for viral upper respiratory tract infection and increased antibiotic use for pneumonia. For the indicators "\% viral upper respiratory tract infections treated with antibiotics" and "\% patients treated with antibiotics", where reduced use is better use, the sign of the calculated result in Table 3 has been changed, so that its positive sign is associated with an increased use of appropriate antibiotics.

All original studies (except \#43) included statistical hypothesis testing with 0.05 or 0.001 level of significance, while none constructed confidence intervals around the results. As illustrated in Table 2, significant differences between intervention and control groups had been reported in the studies where we calculated at least $8 \%$ improvement.

We found out that the indicator "percentage of 
Table 2: Effectiveness of the included interventions.

\begin{tabular}{|c|c|c|}
\hline Ref & Intervention types / components & AB use indicators / Impact size* \\
\hline$\# 40$ & $\begin{array}{l}\text { Provider and consumer education, } \\
\text { Economic strategies and EML } \\
\text { Training workshops, printed materials } \\
\text { for doctors \& consumers, STGs, } \\
\text { limiting drug treatment cost for single } \\
\text { disease, EML creation }\end{array}$ & \begin{tabular}{|l|} 
\% patients prescribed $\mathrm{AB}$ \\
Control base line: $38.5 \%$, post1: $37 \%$, \\
Difference: $-1.5 \%$ (no stat. significance, $p>0.005)^{\star *}$ \\
Intervention baseline: $37.7 \%$, post $1: 33.7 \%$, \\
Difference: $-4 \%$ (no stat. significance, $p>0.005)^{* *}$ \\
Intervention impact: $(-4 \%)-(-1.5 \%)=-2.5 \%$ \\
\end{tabular} \\
\hline $\begin{array}{l}\# 41 \\
\text { (a) }\end{array}$ & $\begin{array}{l}\text { Consumer education } \\
\text { Public education consisting of group } \\
\text { discussions and talks with ARI } \\
\text { prevention guidelines and pamphlets, } \\
\text { Dissemination of educational } \\
\text { materials by health staff. }\end{array}$ & $\begin{array}{l}\text { \% URTI cases treated with AB } \\
\text { Control base line: } 19.6 \% \text {, post1: } 20.3 \% \text {, Difference: } \\
0.7 \% \\
\text { Intervention baseline: } 11.4 \% \text {, post } 1: 12.3 \%, \\
\text { Difference: } 0.9 \% \\
\text { (Statist. significant difference between control and } \\
\text { intervention groups for post } 1, p=0.004)^{* *} \\
\text { Intervention impact: } 0.9 \%-0.7 \%=0.2 \%\end{array}$ \\
\hline $\begin{array}{l}\# 41 \\
\text { (b) }\end{array}$ & $\begin{array}{l}\text { Provider education } \\
\text { and Manag erial strategy } \\
\text { Refresher training course for family } \\
\text { physicians: prelim test of ARI } \\
\text { knowledge, video on ARI clinical } \\
\text { exploration, audio-visual slide } \\
\text { pre sentation on treatment, group } \\
\text { discussion of guidelines, follow-up } \\
\text { session, advisory visits }\end{array}$ & $\begin{array}{l}\text { \% URTI cases treated with AB } \\
\text { Control base line:19.6\%, post } 1: 20.3 \%, \text { Difference: } \\
0.7 \% \\
\text { Intervention baseline: } 20.6 \%, \text { post } 1: 11.7 \%, \\
\text { Difference: }-8.9 \% \\
\text { (Statistically significant difference between control } \\
\text { and intervention groups for post } 1)^{\star *} \\
\text { Intervention impact: }(-8.9 \%)-0.7 \%=-9.6 \%\end{array}$ \\
\hline $\begin{array}{l}\# 41 \\
\text { (c) }\end{array}$ & $\begin{array}{l}\text { Provider \& consumer education and } \\
\text { Managerial strategy } \\
\text { Refresher training course for family } \\
\text { doctors and Public education as in } \\
\# 41 a, b)\end{array}$ & $\begin{array}{l}\text { \% URTI cases treated with AB } \\
\text { Control baseline: } 19.6 \%, \text { post1: } 20.3 \% \text {, Difference: } \\
0.7 \% \\
\text { Intervention baseline: } 26.6 \% \text {, post } 1: 7.8 \%, \\
\text { Difference: }-18.8 \% \\
\text { (Statistically significant difference between control } \\
\text { and intervention groups for post } 1)^{* *} \\
\text { Intervention impact: }(-18.8 \%)-0.7 \%=-19.5 \%\end{array}$ \\
\hline$\# 42$ & $\begin{array}{l}\text { Provider education } \\
\text { and Managerial strategy } \\
\text { Quality Assurance Project: facility- } \\
\text { based quality improvement teams, } \\
\text { standards communication, refresher } \\
\text { training, strengthening hosp pharmacy } \\
\text { committees, monitoring of compliance } \\
\text { indicators, users'committee }\end{array}$ & $\begin{array}{l}\text { \% pneumonia cases treated with appropriate AB } \\
\text { Control baseline:65\%, post } 1: 75 \%, \text { Difference: } 10 \% \\
\text { Intervention baseline: } 85 \%, \text { post } 1: 100 \%, \\
\text { Difference: } 15 \% \\
\text { Intervention impact: } 15 \%-10 \%=5 \% \\
\text { (No significant difference between control and } \\
\text { intervention groups)** }\end{array}$ \\
\hline$\# 43$ & $\begin{array}{l}\text { Provider education } \\
\text { Small group interactive leaming in } \\
\text { CME with trainers, followed with self- } \\
\text { learning materials sent to participants } \\
\text { later. Control group had same CME } \\
\text { with no follow-up. }\end{array}$ & $\begin{array}{l}\% \text { patients prescribed } \mathrm{AB} \\
\text { Control base line: } 59 \%, \text { post } 1: 60 \%, \\
\text { Difference: } 1 \% \\
\text { Intervention baseline: } 61 \% \text {, post } 1: 63 \% \text {, } \\
\text { Difference: } 2 \% \\
\text { Intervention impact: } 2 \%-1 \%=1 \% \\
\end{array}$ \\
\hline$\overline{744}$ & $\begin{array}{l}\text { Provider education } \\
\text { CME structures short course in } \\
\text { improving rational AB prescribing } \\
\text { behavior with presentations, case } \\
\text { discussion, questions \& answers, } \\
\text { panel discussion \& evaluation. }\end{array}$ & $\begin{array}{l}\text { \% patients prescribed } \mathrm{AB} \\
\text { Control baseline: } 71.4 \%, \text { post1: } 74.8 \%, \\
\text { Difference: } 3.4 \% \\
\text { Intervention baseline: } 66.8 \% \text {, post1: } 66.1 \% \text {, } \\
\text { Difference:-0.7\% } \\
\text { Intervention impact: }(-0.7 \%)-3.4 \%=-4.1 \% \\
\text { (not statistically significant) }{ }^{* *}\end{array}$ \\
\hline$\# 45$ & \begin{tabular}{|l|} 
Provider education \\
Interactive group discussion
\end{tabular} & $\begin{array}{l}\text { \% patients prescribed } \mathrm{AB} \\
\text { Control baseline: } 40.8 \%, \text { post } 1: 37.2 \%, \\
\text { Difference:-3.6\% } \\
\text { Intervention baseline: } 40.8 \%, \text { post1: } 38.9 \%, \\
\text { Difference:-1.9\% } \\
\text { Intervention impact: }(-1.9 \%)-(-3.6 \%)=1.7 \% \\
\text { (not statistically significant) }{ }^{\star *}\end{array}$ \\
\hline \#46 & $\begin{array}{l}\text { Provider education } \\
\text { Development \& dissemination of } \\
\text { evidence-ba sed leaflet summary } \\
\text { about appropriate URTI management } \\
\text { and academic detailing in form of a } \\
\text { single one-to-one meeting with } \\
\text { opinion leader. Summaries given to } \\
\text { medical officers under opinion leader. }\end{array}$ & $\begin{array}{l}\text { \% patients prescribed AB } \\
\text { Control baseline: } 14.1 \%, \text { post1: } 13.9 \%, \\
\text { Difference } 0.2 \% \\
\text { Intervention baseline: } 14.3 \%, \text { post1: } 11 \% \\
\text { Difference: }-3.3 \% \\
\text { Intervention impact: }(-3.3 \%)-(0.2 \%)=-3.1 \% \\
\text { (not statistically significant, } p=0.942)^{* *} \\
\% \text { URTI cases treated with AB } \\
\text { Control baseline: } 26 \%, \text { post1: } 28.5 \% \\
\text { Difference: }-2.5 \% \\
\text { Intervention baseline: } 27.7 \%, \text { post3: } 16.6 \% \\
\text { Difference: }-11.1 \% \\
\text { Intervention impact: }(-11.1 \%)-(-2.5 \%)=-8.6 \% \\
\text { statistically significant, } p<0.001)^{* *}\end{array}$ \\
\hline$\# 47$ & $\begin{array}{l}\text { Provider education } \\
\text { and Managerial strategy } \\
\text { An interactive educational and } \\
\text { managerial intervention on } \\
\text { ynopharyngitis treatment, using an } \\
\text { interactive educational workshop and } \\
\text { a managenal peer review committee. }\end{array}$ & $\begin{array}{l}\text { \% patients prescribed } \mathrm{AB} \\
\text { Control baseline: } 96.2 \%, \text { post } 1: 93.4 \% \text {, Difference: } \\
-2.8 \% \\
\text { Intervention baseline: } 84.3 \%, \text { post1: } 60 \%, \\
\text { Difference: }-24.3 \% \\
\text { Intervention impact: }-24.3 \%-(-2.8 \%)=-21.5 \% \\
\text { (statistically significant, } p<0.05)^{* *} \\
\% \text { URTI cases treated with } \mathrm{AB} \\
\text { Control baseline: } 77.6 \%, \text { post2: } 76.9 \%, \\
\text { Difference: }-0.7 \% \\
\text { Intervention baseline: } 70.4 \%, \text { post1: } 52.1 \%, \\
\text { Difference:- } 18.3 \%, \\
\text { Intervention impact: }(-18.3 \%)-(-0.7 \%)=-17.6 \% \\
\text { (statistically significant, } p<0.05)^{* *}\end{array}$ \\
\hline
\end{tabular}

AB - Antibiotics, ARI - Acute Respiratory Infection, URTI -Upper Respiratory Tract Infection, PHC - Primary Healthcare Centre, MD - Medical Doctors, EML - Essential Medicines List, STG - Standard Treatment Guidelines, CME - Continuing Medical Education. *-For the intervention impact, improved antibiotic use is reflected by reduced antibiotic use in general and for URTI (negative value) and increased antibiotic use in pneumonia (positive value). ** - Information extracted from the original studies.

patients prescribed antibiotics" was reduced only by multifaceted interventions focusing on specific diseases (Table 3). For instance, the intervention in study \#47 targeted ARI using multiple strategies (peer review committees), and reduced the indicator by $21.5 \%$. By contrast, interventions with no focus on specific diseases (studies \# 40,43-46) achieved low or no impact on this indicator (-1.7\% to $4.1 \%)$. Four of them included only educational interventions (studies \#43-46), while the intervention in study \#40 was multifaceted. However, even the multifaceted intervention did not reduce the indicator (-2.5\%), as it did not target any specific disease.

Similarly, the indicator "percentage of URTI treated with antibiotics" was reduced only by multifaceted interventions targeting specific diseases (Table 3). Interventions that combined educational and managerial interventions in the studies \#46 (academic detailing) and \#47 (interactive educational workshop with peer review

Table 3: Summary of the interventions and their effect size.

\begin{tabular}{|c|c|c|c|c|}
\hline Ref & Country & Intervention types & $\begin{array}{l}\text { AB use indicators } \\
\text { and Disease type }\end{array}$ & $\begin{array}{l}\text { Impact } \\
\text { size* }^{*}\end{array}$ \\
\hline$\# 45$ & Iran & Provider education & $\begin{array}{l}\text { \% patients prescribed } A B \\
\text { (all diseases) }\end{array}$ & $-1.7 \%$ \\
\hline$\# 43$ & Iran & $\begin{array}{l}\text { Provider education } \\
\text { with self-learning } \\
\text { materials sent after } \\
\text { the training }\end{array}$ & $\begin{array}{l}\% \text { patients prescribed } \mathrm{AB} \\
\text { (all diseases) }\end{array}$ & $-1 \%$ \\
\hline $\begin{array}{l}\# 41 \\
a\end{array}$ & Cuba & Consumer Education & $\begin{array}{l}\text { \% URTI cases treated with } \\
A B\end{array}$ & $-0.2 \%$ \\
\hline$\# 40$ & China & $\begin{array}{l}\text { Provider \& consumer } \\
\text { education / Printed } \\
\text { materials / Economic } \\
\text { strategy and EML }\end{array}$ & $\begin{array}{l}\text { \% patients prescribed } A B \\
\text { (all diseases) }\end{array}$ & $2.5 \%$ \\
\hline$\# 44$ & Iran & Provider education & $\begin{array}{l}\text { \% patients prescribed } \mathrm{AB} \\
\text { (all diseases) }\end{array}$ & $4.1 \%$ \\
\hline$\# 42$ & Ecuador & $\begin{array}{l}\text { Provider education / } \\
\text { Printed material / } \\
\text { Group process and } \\
\text { managerial strategies } \\
\text { (hospital pharmacy } \\
\text { committees) } \\
\end{array}$ & $\begin{array}{l}\% \text { pneumonia cases treated } \\
\text { with appropriate } A B\end{array}$ & $5 \%$ \\
\hline$\# 46$ & Malaysia & \begin{tabular}{|l|} 
Provider \\
education/Printed \\
materials
\end{tabular} & $\begin{array}{l}\text { \% patients prescribed } A B \\
\text { (all diseases) } \\
\text { \% URTI cases treated with } \\
A B\end{array}$ & $\begin{array}{l}3.1 \% \\
8.6 \% \text { ** }\end{array}$ \\
\hline $\begin{array}{l}\# 41 \\
\mathrm{~b}\end{array}$ & Cuba & $\begin{array}{l}\text { Provider } \\
\text { education/Printed } \\
\text { materials/Managerial } \\
\text { strategy (advisory } \\
\text { visits) }\end{array}$ & $\begin{array}{l}\text { \% URTI cases treated with } \\
A B\end{array}$ & $9.6 \% \%^{* * *}$ \\
\hline $\begin{array}{l}\# 41 \\
\mathrm{c}\end{array}$ & Cuba & $\begin{array}{l}\text { Provider \& consumer } \\
\text { education /Printed } \\
\text { material//Managerial } \\
\text { strategy (advisory } \\
\text { visits) } \\
\end{array}$ & $\begin{array}{l}\text { \% URTI cases treated with } \\
A B\end{array}$ & $19.5 \%^{* * \star}$ \\
\hline$\# 47$ & Mexico & $\begin{array}{l}\text { Provider education / } \\
\text { Printed materials / } \\
\text { Group process and } \\
\text { managerial strategies } \\
\text { (peer review } \\
\text { committees) }\end{array}$ & $\begin{array}{l}\text { \% URTI cases treated with } \\
A B \\
\text { \% patients prescribed } A B \\
\text { (all diseases) }\end{array}$ & $\begin{array}{l}17.6 \% \%^{* *} \\
21.5 \% \text { ** }\end{array}$ \\
\hline
\end{tabular}

* - Intervention effects are expressed positively in comparison with Table 1 where the actual values of effectiveness are expressed. Thus, a decrease (negative value) in antibiotic use in general and for URTI and an increase in antibiotic use for pneumonia represents improved (positive value) use. ** - Statistically significant in original studies. *** - Statistically significant (positive value) use. ${ }^{* *}$ - Statistically significant in original studies. ${ }^{\star * *}$ - Statistically significant
difference between control and intervention groups after the intervention in original studies. 
committees) focusing on ARI, reduced the indicator by 8.6\% and $17.6 \%$ respectively. Likewise, study \#41 examined the impact of three different interventions on the indicator "percentage of URTI treated with antibiotics". First, the single 'Consumer education' intervention did not reduce the indicator (-0.2\%). Second, the multifaceted intervention consisting of 'Provider education', 'Printed materials' and 'Managerial strategy' (refresher training course, group discussion of guidelines, follow-up session, and advisory visits) had an impact of $9.6 \%$. When 'Consumer education' was added to the latter combination, the overall impact increased to $19.5 \%$.

Lastly, we investigated the intervention effectiveness on the indicator "appropriate antibiotic prescribing in pneumonia", However, it was difficult to draw any conclusions as the indicator was used only in study \#42. This intervention targeted a specific disease (ARI) and used combined intervention strategies. Both control and the intervention groups had high baseline rates and achieved low impact effect (5\%), which was reported as statistically not significant.

\section{Discussion}

We identified only eight studies of acceptable quality that evaluated interventions for improving antibiotic prescribing in 54 UMICs over a period of 20 years. This small body of evidence may not be sufficient to provide comprehensive knowledge on the subject. However, some conclusions can still be drawn about effectiveness of different strategies.

It is important to note that the particular group of UMICs was selected in order to learn about health interventions applicable to the Republic of Macedonia. However, WB classification by income level does not imply that countries in the same group experience similar health care system development [36]. To overcome some of the context diversity, search criteria included only interventions that affected the prescribing by a homogenous group of medical doctors. It is disappointing that no valid study has been detected from Eastern Europe (WB region of Europe/Central Asia) because of the healthcare setting similarity with the Republic of Macedonia [49]. As the country is European Union candidate, it is recommended to expand future research by including European high-income countries.

Our results show that interventions were predominantly educational in nature, and conducted only in the public sector. In general, the intervention effects were similar to findings from other systematic reviews on antibiotic use in developed and developing countries [32, 34, 35]. Results suggest that interventions with low or no impact on antibiotic prescribing employ only educational components with no specific disease focus. Multi-faceted interventions, involving educational and managerial components, such as education for both medical doctors and patients combined with advisory visits or peer review committees improved the antibiotic prescribing in primary healthcare. Similarly, interventions focusing only on specific diseases have great potential for improving antibiotic prescribing practices.

In order to develop targeted intervention strategies, it is essential to generate more reliable data through research. Particular areas that need further improvement include: conducting more well designed evaluations of interventions, increasing the reporting quality of the results by adding their significance and degree of variability, and publishing study findings.

Conclusions: The issue of inappropriate use of antibiotics is of global concern, because of its association with increased antibiotic resistance. Various interventions have been carried out with the aim of improving antibiotic prescribing practice. This review has shown that only limited evidence with acceptable quality on interventions evaluation in UMICs exists. While our results emphasize the important role of multifaceted interventions in the public sector, much still remains unexplored about practices in the private sector. Countries and international organizations need to be more committed to monitor antibiotic prescribing regularly and to conduct well designed evaluations of interventions.

\section{References}

1. European Centre for Disease Prevention and Control. Antimicrobial resistance. European Centre for Disease Prevention and Control, 2011. www.ecdc.europa.eu/en/ healthtopics/antimicrobial_resistance/Pages/index.aspx. Accessed on 28 November 2011.

2. Holloway K, van Dijk L. Rational Use of Medicines. In: The World Medicines Situation Report 2011. World Health Organization $3^{\text {rd }}$ Edition, Geneva 2011. http://apps.who.int/ medicinedocs/documents/s18064en/s18064en.pdf.

3. World Health Organization. Medicines use in primary care in developing and transitional countries: Fact book summarizing results from studies reported between 1990 and 2006. World Health Organization, Geneva, 2009. http:// w w w. who.int/medicines/publications/ primary_care_8April09.pdf. 
4. De Melker RA,Kuyvenhoven MM. Management of upper respiratory tract infections in Dutch family practice. J Fam Pract. 1994;38:353-7.

5. McCaig LF, Besser RE, Hughes JM. Antimicrobial drug prescription in ambulatory care settings, United States, 19922000 [Published correction appears in Emerg Infect Dis 2003;9:609]. Emerg Infect Dis. 2003;9:432-7.

6. Arroll B, Kenealy T. Antibiotics for the common cold. Cochrane Database Syst Rev. 2000;(2):CD000247.

7. Del Mar CB, Glasziou PP, Spinks AB. Antibiotics for sore throat. Cochrane Database Syst Rev. 2000;(4):CD000023.

8. Becker L, Glazier R, Mclsaac W, Smucny J. Antibiotics for acute bronchitis. Cochrane Database Syst Rev 2000;(2):CD000245.

9. Glasziou PP, Del Mar CB, Sanders SL, Hayem M Antibiotics for acute otitis media in children. Cochrane Database Syst Rev. 2004;(1):CD000219.

10. Williams JW, Aguilar C, Makela M, Cornell J, Hollman DR, Chiquette $\mathrm{E}$, et al. Antibiotics for acute maxillary sinusitis. Cochrane Database Syst Rev. 2003;(2):CD000243.

11. Goossens H, Ferech M, Vander Stichele R, Elseviers M. ESAC Project Group. Outpatient antibiotic use in Europe and association with resistance: a cross-national database study. Lancet. 2005; 365: 579-587.

12. Van Roosmalen MS et al. Antibiotic prescribing in primary care: First choice and restrictive prescribing are two different traits. Qual Saf Health Care. 2007;16(2):105-109.

13. Arroll B, Kenealy T. Antibiotics for the common cold. Cochrane Database Syst Rev. 2000;(2):CD000247

14. Mainous AG 3rd, Hueston WJ. The cost of antibiotics in treating upper respiratory tract infections in a Medicaid population. Arch Fam Med. 1998; 7: 45-49

15. Goossens H. Antibiotic consumption and link to resistance. Clin Microbiol Infect. 2009;15(Suppl. 3):S12-S15.

16. World Health Organization. WHO Antimicrobial resistance. Fact sheet N¹94 February 2011. World Health Organization, Geneva, 2011. www.who.int/mediacentre/factsheets/fs194/en/

17. Kaplan W, Laing R. Priority medicines for Europe and the world. World Health Organization, Geneva,, 2004. http:// hinfo.humaninfo.ro/gsdldata/collect/medicinedocs/index/ assoc/s14208e/s14208e.pdf.

18. Goossens $\mathrm{H}$ et al. European Surveillance of Antimicrobial Consumption Project Group. Comparison of outpatient systemic antibacterial use in 2004 in the United States and 27 European countries. Clin Infect Dis 2007, 44:1091-1095.

19. Jelinski S, Parfrey P, Hutchinson J. Antibiotic utilisation in community practices: guideline concurrence and prescription necessity. Pharmacoepidemiol Drug Saf 2005; 14: 319-26.

20. World Health Organization. World Health Assembly Resolution 58.27. Improving the containment of antimicrobial resistance. World Health Organization, Geneva, 2005. http:// apps.who.int/gb/ebwha/pdf_files/WHA58/WHA58_27-en.pdf.

21. World Health Organization. World Health Assembly Resolution 60.16. Progress in the rational use of medicine. World Health Organization, Geneva, 2007. http://apps.who.int/ gb/ebwha/pdf_files/WHA60/A60_R16-en.pdf.

22. World Health Organization. WHO Global Strategy for Containment of Antimicrobial Resistance. World Health Organization, Geneva, 2001. www.who.int/drugresistance/ WHO_Global_Strategy_English.pdf.

23. European Commission. Action plan against the rising threats from Antimicrobial Resistance. European Commission, Brussels, 2011. http://ec.europa.eu/dgs/health_consumer/ docs/communication_amr_2011_748_en.pdf.

24. Centers for Disease Control and Prevention. Treatment Guidelines for Upper Respiratory Tract Infections. Centers for Disease Control and Prevention 2006. www.aafp.org/afp/2006/ 0915/p956.pdf.

25. National Health Service, UK. Respiratory tract infections - antibiotic prescribing. National Institute for Health and Clinical Excellence. NHS 2008. www.nice.org.uk/nicemedia/pdf/ CG69FullGuideline.pdf.

26. World Health Organization. Acute Respiratory Infections (ARI). Case Management Charts. World Health Organization, Geneva, 1991.

27. World Health Organization. A Manual for the Treatment of Diarrhoea. World Health Organization, Geneva, 1990.

28. World Health Organization. Promoting rational use of medicines: core components. WHO Policy Perspectives of Medicines, no. 5. World Health Organization, Geneva, 2002.

29. Holloway KA. Combating Inappropriate Use of Medicines. Expert Rev Clin Pharmacol. 2011;4(3):335-348.

30. World Health Organization. Medicines: rational use of medicines. Fact sheet $\mathrm{N}^{\circ} 338$, World Health Organization, Geneva, 2010. www.who.int/mediacentre/factsheets/fs338/en/ index.html.

31. Belongia EA, Schwartz B. Strategies for promoting judicious use of antibiotics by doctors and patients. BMJ. 1998;317:66871.

32. Arnold SR, Evans M, Straus SE. Interventions to improve antibiotic prescribing practices in ambulatory care (Protocol for a Cochrane Review). Cochrane Library; Issue 1, 2003. Oxford: Update Software.

33. Gross PA, Pujat D. Implementing practice guidelines for appropriate antimicrobial usage. A systematic review. Med 
Care. 2001;39:II55-69.

34. Welschen I, Kuyvenhoven MM, Hoes AW, et al. Effectiveness of a multiple intervention to reduce antibiotic prescribing for respiratory tract symptoms in primary care: randomised controlled trial. BMJ. 2004; 21; 329 (7463) :431.

35. World Health Organization. Interventions and strategies to improve the use of antimicrobials in developing countries. Drug Management Program. WHO/CDS/CSR/DRS/2001. World Health Organization, Geneva, 2001. www.who.int/csr/ resources/publications/drugresist/ WHO_CDS_CSR_DRS_2001_9/en/

36. World Bank. Country and Lending Groups. http:// data.worldbank.org/about/country-classifications/country-andlending-groups\#Upper_middle_income. Accessed on 26 November 2011.

37. International Network for the Rational Use of Drugs. INRUD Medicines Use bibliography. http://www1.inrud.org:81/ rmwp?\&func=advSearch.

38. World Health Organization. How to investigate drug use in health facilities: Selected drug use indicators. (DAP Research Series $\mathrm{N}^{\circ}$ 7. WHO/DAP/93.1). World Health Organization, Geneva, 1993.

39. World Health Organization. Integrated Management of Childhood Illness. World Health Organization, Geneva, 1999. http://www.idpas.org/58relatedprograms1.html.

40. Yu-qin Wang, Lang Zhang, et al . Multi-centre indicator intervention research on rational drug use, Xuanwu Hospital Group and Centre for Drug Re-evaluation of State Drug Administration and Ministry of Health, Beijing, China, 2007.

41. Gonzalez Ochoa E, Armas Perez L, Bravo Gonzalez JR,
Cabrales Escobar J, Rosales Corrales R, Abreu Suarez G. Prescription of antibiotics for mild acute respiratory infections in children. Bull Pan Am Health Org, 1996; 30:106-117.

42. Hermida J, Robalino ME. Increasing compliance with maternal and child care quality standards in Ecuador. Int J Qual Health Care. 2002;14(suppl 1): 25-34.

43. Esmaily HM, Silver I, Shiva S, Garjani A, et al., Can rational prescribing be improved by an outcome-based educational approach? A randomised trial completed in Iran; J Contin Educ Health Prof. 2010;30(1):11-18.

44. Mohagheghi MA, Mosavi-Jarrahi A, Khatemi-Moghaddam M, Afhami S, Khodai S, Azemoodeh O. Community-based outpatient practice of antibiotics use in Tehran. Pharmacoepidemiol Drug Saf. 2005;14: 135-138.

45. Garjani A, Salimnejad M, Shamsmohamadi M, Baghchevan V, Vahidi RG, Maleki-Dijaz N, Rezazadeh H. Effect of interactive group discussion among physicians to promote rational prescribing. East Mediterr Health J, 2009;15(2).

46. Teng $C L$ et al. Modifying antibiotic prescribing: the effectiveness of academic detailing plus information leaflet in a Malaysian primary care setting. Med J Malaysia. 2006;61(3): 323-331.

47. Perez-Cuevas R, Guiscafre H, Munoz O, Reyes H, Tome $\mathrm{P}$, Libreros $\mathrm{V}$ et al. Improving physician prescribing patterns to treat rhinopharyngitis. Intervention strategies in two health systems of Mexico. Soc Sci and Med. 1996; 42(8):1185-1194.

48. World Health Organization. The Former Yugoslav Republic of Macedonia Health system review on Health Systems and Policies. Health Systems in Transition 2006; 8(2):1-98. The European Observatory on Health Systems and Policies, World Health Organization, 2006. 